\title{
Elastic-plastic Analysis and Experimental Study on the Simply Supported Bi-modulus Graphite Beam
}

\author{
Wen-Juan $Y_{A O}^{1,2, a}$, Jin-Ling GAO ${ }^{1,2}$, Yang YANG ${ }^{1}$ and Yu-Ting ZHOU ${ }^{1}$ \\ ${ }^{1}$ Shanghai University, Department of Civil Engineering, 200444 Shanghai, China \\ ${ }^{2}$ Shanghai Institute of Applied Mathematics and Mechanics, 200072 Shanghai, China
}

Keywords : Graphite, Bi-modulus, Elastic-plastic model, Material test, Structural experiment.

\begin{abstract}
Based on bi-modulus theory and experimental test, the elastic-plastic model of bending bi-modulus graphite beam is established in this paper. According to the results of material test, the graphite is demonstrated to be a bi-modulus material with the ratio of compression modulus to tension modulus being approximately 1.41. By investigating the mechanical behavior of pure bending graphite beam, the deflection law of the neutral axis towards the load and the $\mathrm{N}-\varepsilon$ curve are obtained. It shows that the experimental results are in accordance with the theoretical elastic-plastic model of the bending bi-modulus beam established in this paper, which verifies the accuracy and rationality of the theoretical model.
\end{abstract}

\section{Introduction}

It is shown that many materials in engineering have different modulus in tension and compression, which are defined as bi-modulus materials, such as concrete in civil engineering [1], metal in mechanical engineering [2], graphite in nuclear engineering [3] and plastic in aerospace engineering [4]. With the progression of science and technology and the widely application of novel materials, the research on mechanical characteristics of bi-modulus materials has been a new trend. Graphite is such a kind of bi-modulus material applied to be many devices like the electrode in the steelmaking process using electric arc method, the graphite annulus used to mechanical sealing. Because of the excellent mechanical and thermal property as well as the irradiation resistance property, graphite is also applied in the construction of nuclear reactor. Recently, graphite has been studied in depth [5-9].

However, few researches have been conducted considering the bi-modulus property of the material. In this paper, Material tests are firstly conducted and the ratio of compression modulus to tension modulus of graphite is investigated. Afterwards, structural experiment of the pure bending bi-modulus graphite beam is then conducted to obtain the deflection law of the neutral axis in the

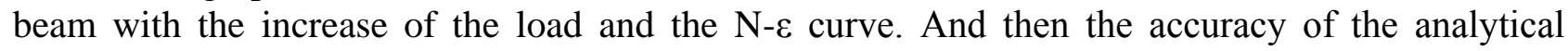
solution of elastic mechanical response of bi-modulus beam proposed in [10] is verified. Simultaneously, the whole process elastic-plastic analysis of the simply supported bi-modulus beam is conducted by introducing bi-modulus theory. The results show that the position of neutral axis and the maximum ratio of compressive stress to tensile stress during the elastic process are only related to the ratio of compressive modulus to tensile modulus but unrelated to the stress state. Finally, the accuracy of the elastic-plastic analysis considering bi-modulus property of materials is demonstrated by comparing the theoretical results with the experimental results of structural test of the simply supported graphite beam.

\section{Material Tests}

Material tests are designed to investigate the mechanical properties of MSL82 graphite. The graphite samples are divided into 4 cylinder-shape specimens with the diameter of cross section $\Phi=10 \mathrm{~mm}$ and the length $\mathrm{L}=50 \mathrm{~mm}$, and another 4 cylinder-shape specimens with the diameter of cross-section $\Phi=10 \mathrm{~mm}$ and the length $\mathrm{L}=200 \mathrm{~mm}$. The testes are performed by using an electronic universal testing machine (CMT5306) and an electronic universal testing machine (WDW-E100). The testing contents in detail include the ultimate tensile strength, the ultimate compressive strength, 
the tensile modulus, the compressive modulus and the ratio of compressive modulus to tensile modulus Ec/Et. The results are tabulated in Table 1.

Therefore, the results show that the graphite material has the distinct bi-modulus property. Ec/Et is approximately 1.41 .

\section{Elastic Analysis}

\section{Structural Experiment}

Table 1. Material Test results of Graphite (MSL82).

\begin{tabular}{|c|c|c|c|c|c|}
\hline $\begin{array}{c}\text { Specimen } \\
\text { number }\end{array}$ & $\begin{array}{c}\text { Ultimate tensile } \\
\text { strength(Mpa) }\end{array}$ & $\begin{array}{c}\text { Ultimate } \\
\text { compressive } \\
\text { strength(Mpa) }\end{array}$ & $\begin{array}{c}\text { Tensile } \\
\text { modulus } \\
(\mathrm{Gpa})\end{array}$ & $\begin{array}{c}\text { Compressive } \\
\text { modulus(Gpa) }\end{array}$ & $E_{\mathrm{c}} / E_{t}$ \\
\hline 1 & 26.70 & 72.76 & 8.62 & 11.90 & 1.38 \\
\hline 2 & 30.70 & 68.37 & 8.59 & 12.32 & 1.43 \\
\hline 3 & 28.77 & 65.02 & 8.84 & 12.58 & 1.42 \\
\hline 4 & 26.70 & 70.95 & 8.73 & 12.26 & 1.40 \\
\hline Mean & 28.22 & 69.28 & 8.70 & 12.27 & 1.41 \\
\hline
\end{tabular}

As shown in Figure 1, the bending tests are conducted by using an electronic universal testing machine (WDW-E100). The configuration and dimensions $(300 \mathrm{~mm} \times 45 \mathrm{~mm} \times 22 \mathrm{~mm}$ ) of the two graphite specimens are shown in Figure 2 and Figure 3. Eight strain foils, marked No.1 to No.8 respectively, are set on each specimen, of which the positions are shown in Figure 4 and Figure 5.

Therefore, the results show that the graphite material has the distinct bi-modulus property. Ec/Et is approximately 1.41 .

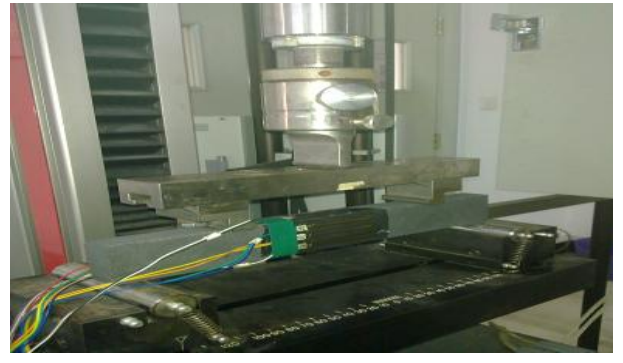

Fig.1. Test device.

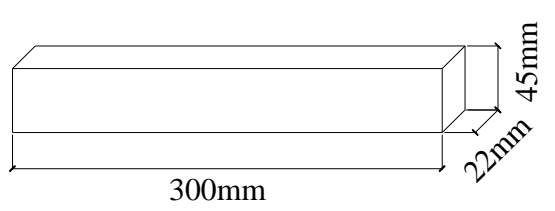

Fig. 3. Specimen size.

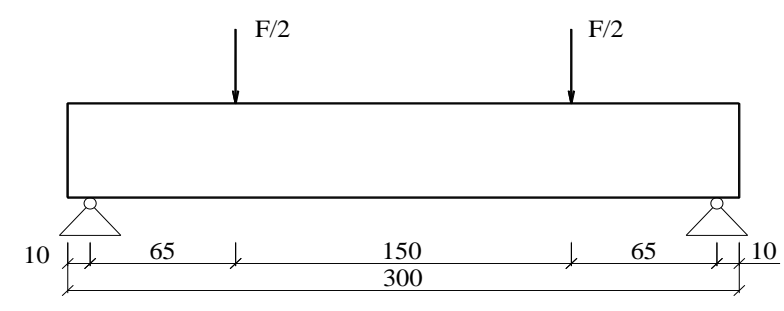

Fig.2. Loading mode.

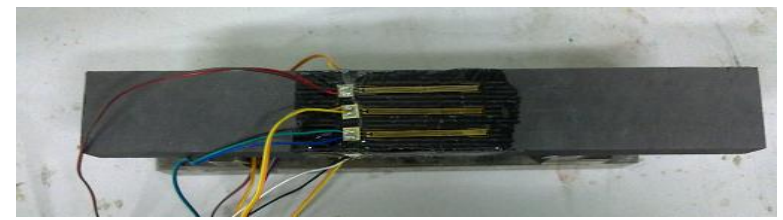

Fig 4. MSL82 graphite specimen with strain foils.
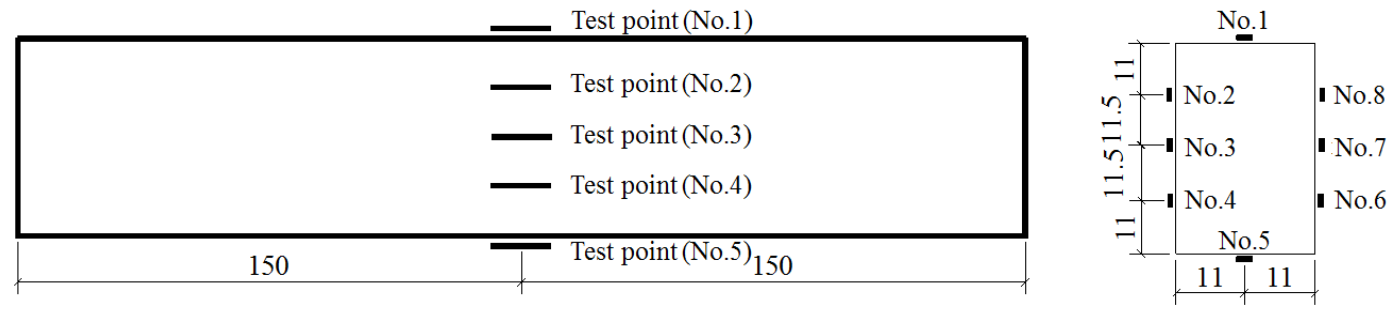

Fig.5. Distribution of strain foils on the specimen. 


\section{Test Results}

\section{Neutral Axis}

Calculate the mean of the strains at the points marked No.2 and No.8, No.3 and No.7, No.4 and No.6, respectively. And then combine the three mean strains with the two strains at the points marked No.1 and No.5 and get the curve. According to the geometric relation, as shown in Figure 6, the curve reflecting the relation between the deflection of the neutral axis $(\delta=\Delta / \mathrm{h})$ and the load can be obtained as Figure 7, where $\varepsilon 1$ is the strain on the top surface of the middle cross section; $\varepsilon 2$ is the strain on the bottom surface of the middle cross section; h1 and h2 are the distance of the neutral axis towards the top surface and the bottom surface, respectively; $h$ is the height of the specimen; $\Delta$ is the distance of the neutral axis towards the middle height of the specimen.
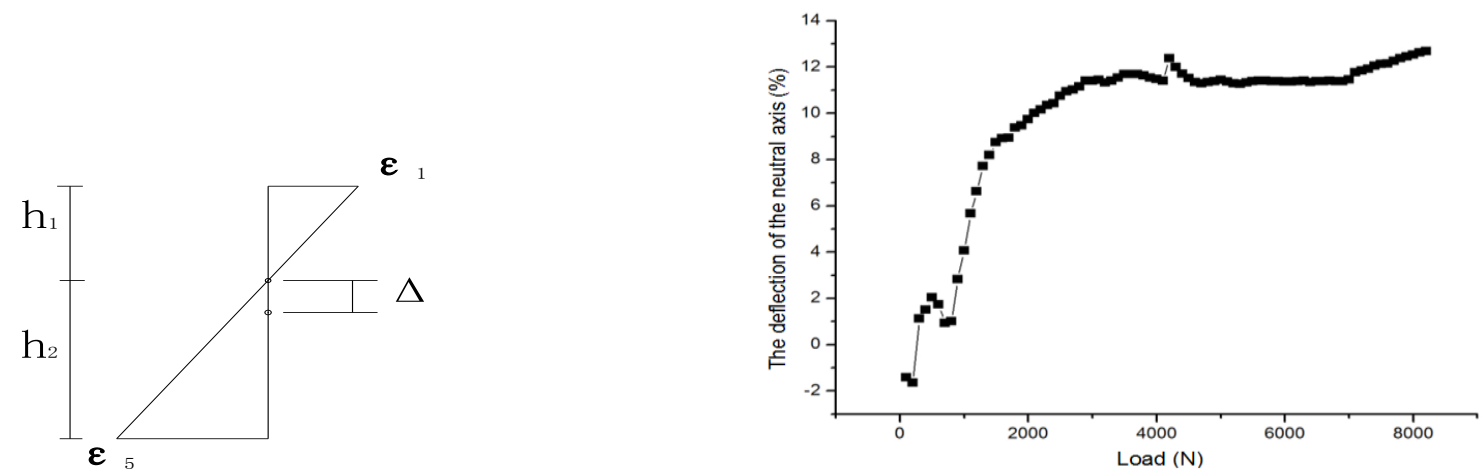

Fig.6. Geometric relation after the change of neutral axis. Fig. 7. The deflection of neutral axis.

\section{Stress-Strain Relation}

Calculate the mean of the test results at the points marked No.2 and No. 8 to be the result of a new point named No.2. Similarly, obtain the test results at the points marked No.4 and No.6 and define a new point as No.4. And then combine the new results at points No.2 and No.4 with the original results at points No.1, No.3 and No.5. Finally, the curves of $\mathrm{N}-\varepsilon$ at each point can be obtained, as shown in Figure 8.

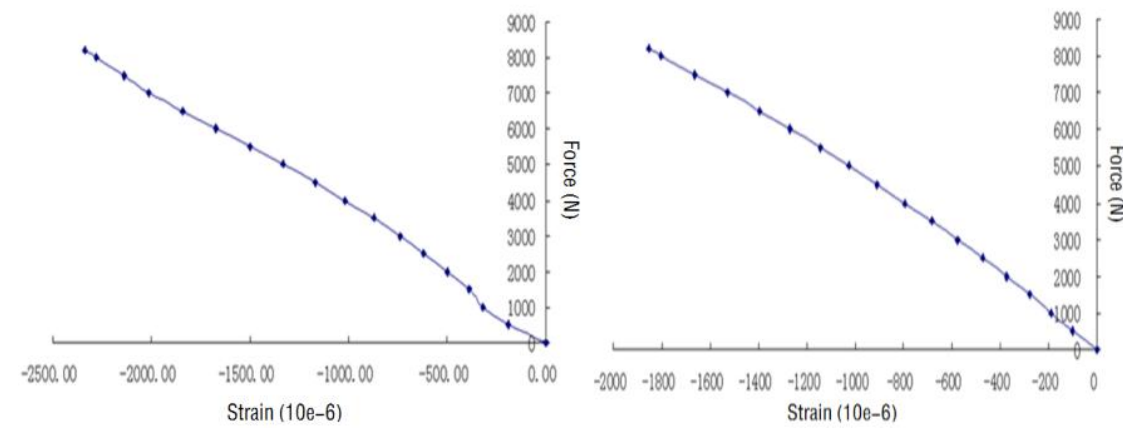

(a). Test point (No.1).

(b). Test point (No.2).

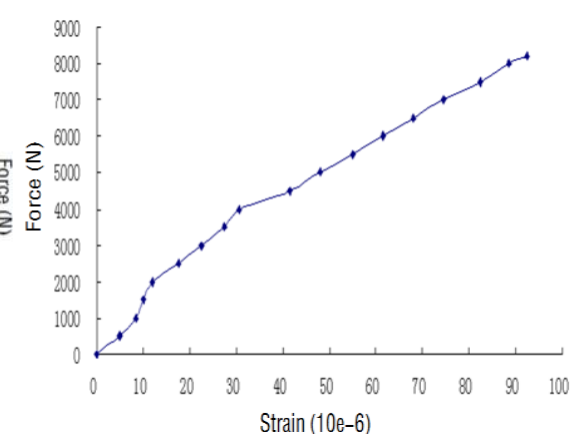

(c). Test point (No.3).

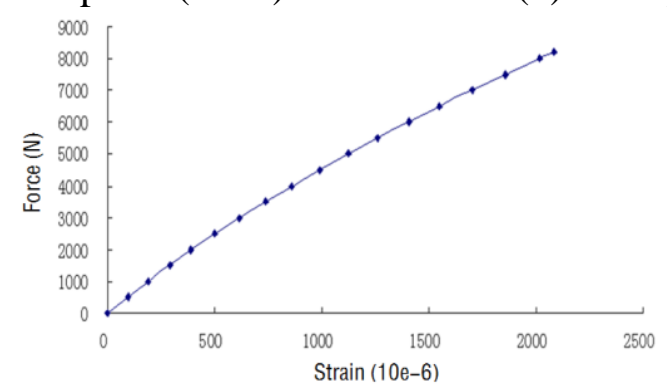

(d). Test point (No.4).

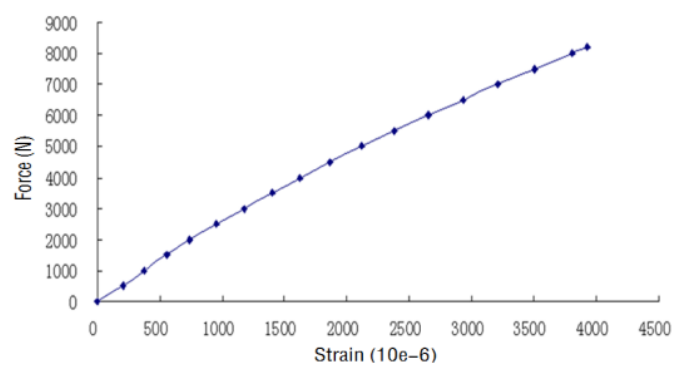

(e). Test point (No.5).

Fig. 8. Stress-strain relation at each point. 


\section{Comparison of Theoretical and Experimental Results}

Eq.(1) is the strain expression of bending beam subjected to the lateral force [10].

$$
\varepsilon_{x}=\frac{3 M\left(\sqrt{E_{t}}+\sqrt{E_{c}}\right)^{2} y}{b h^{3} E_{t} E_{c}}
$$

where Ec and Et are compressive modulus and tensile modulus, respectively; $\mathrm{M}$ is the moment at the middle cross section; $b$ and $h$ are the width and the height of the middle cross section, respectively.

According to the Eq.(1), the theoretical results of strain at each point can be gained and the comparison of theoretical results and experimental results of strain at each point is shown in Figure 9. It is shown that the theoretical results calculated by using the bi-modulus analytical solution proposed in [10] show great accordance with the experimental results in this paper, which verifies the validity of the analytical model in [10].

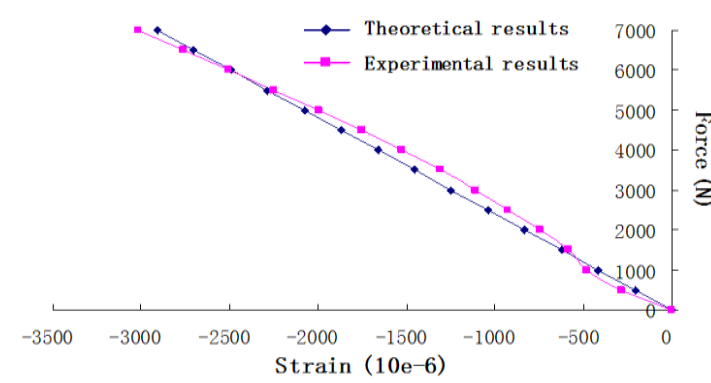

(a). Test point (No.1).

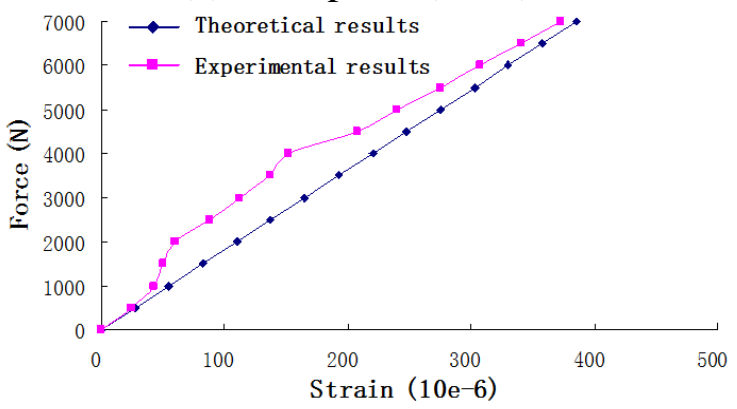

(c). Test point (No.3).

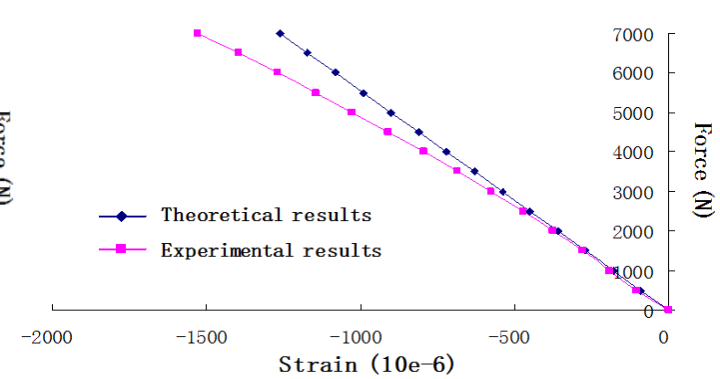

(b). Test point (No.2).

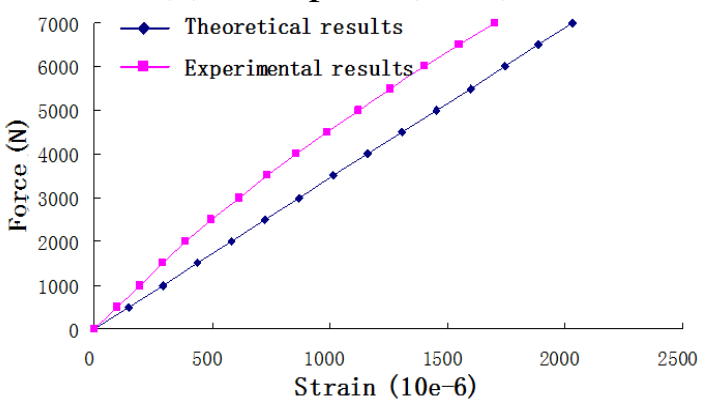

(d). Test point (No.4).

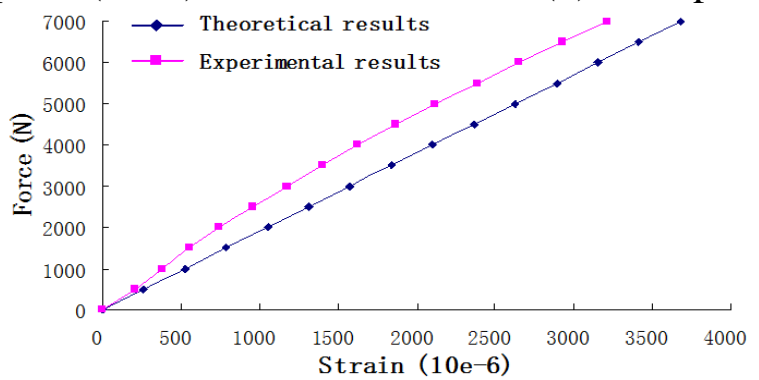

(e). Test point (No.5).

Fig. 9. $\mathrm{N}-\varepsilon$ curve during the elastic period.

\section{Elastic-Plastic Analysis}

\section{Theoretical Analysis}

As shown in Figure 10, a simple supported bi-modulus beam $(2 \mathrm{~L} \times \mathrm{b} \times \mathrm{h})$ is subjected to two symmetrical concentrated loads $\mathrm{P}$. The compressive modulus and tensile modulus are respectively Ec and Et. And the compressive yield coefficient and the tensile yield coefficient are respectively $\mathrm{k} 1$ and $\mathrm{k} 2$. 


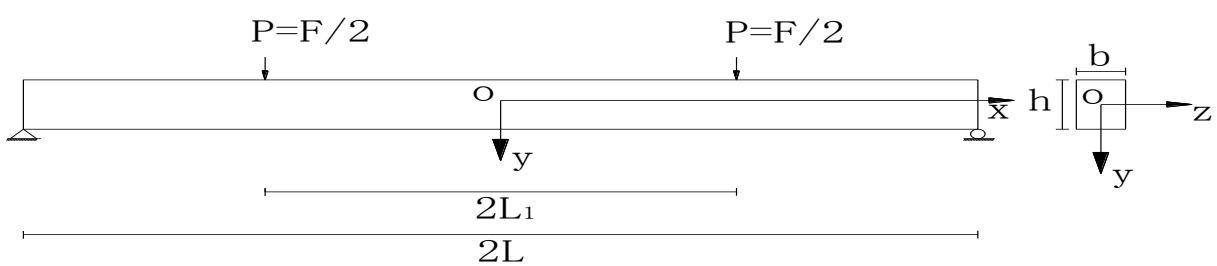

Fig.10. Structural model.

The ratio of tensile modulus to compressive modulus is defined as $\alpha=\mathrm{Et} / \mathrm{Ec}$. Considering the material with $\mathrm{Ec}>\mathrm{Et}(0<\alpha<1)$, then we have

$$
\sigma_{x \max }^{-}>\sigma_{x \max }^{+}
$$

According to the Mises yield criterion, we have

$$
J_{2}=\frac{1}{6}\left[\left(\sigma_{1}-\sigma_{2}\right)^{2}+\left(\sigma_{3}-\sigma_{2}\right)^{2}+\left(\sigma_{1}-\sigma_{3}\right)^{2}\right]=k^{2}
$$

It is worth nothing that the bi-modulus beam investigated is a kind of the plane stress structure and there is no shear stress in the pure bending section. Therefore, we have

$$
\sigma=\sigma_{1} \text { or } \sigma_{2}=\sigma_{3}=0
$$

Correspondingly, the Mises yield criterion can be simplified as

$$
|\sigma|=\sqrt{3} k_{1},|\sigma|=\sqrt{3} k_{2}
$$

At the plastic region, the stresses at points in the compressive region and tensile region are respectively

$$
\sigma=\sqrt{3} k_{1} \text { and } \sigma=\sqrt{3} k_{2}
$$

At the elastic region, the stresses at points in the compressive region and tensile region are respectively

$$
\sigma^{-}=\left(\frac{1+\sqrt{\alpha}}{2 \sqrt{\alpha}}\right)^{2} \frac{M}{I} y \text { and } \sigma^{+}=\left(\frac{1+\sqrt{\alpha}}{2}\right)^{2} \frac{M}{I} y
$$

It is assumed that in elastic region, the height of the tensile region and compressive region are respectively $\xi 1(\mathrm{x})$ and $\xi 2(\mathrm{x})$. The distribution of stress along the height of the cross section is shown in Figure 11. The expressions in detail are written as below

$$
\sigma= \begin{cases}-\sqrt{3} k_{1} & -h_{1} \leq y \leq-\xi_{1} \\ \frac{\sqrt{3} k_{1} y}{\xi_{1}} & -\xi_{1} \leq y \leq 0 \\ \frac{\sqrt{3} k_{2} y}{\xi_{2}} & 0 \leq y \leq \xi_{2} \\ \sqrt{3} k_{2} & \xi_{2} \leq y \leq h_{2}\end{cases}
$$

According the continuity of stress at the inflection points, we have 


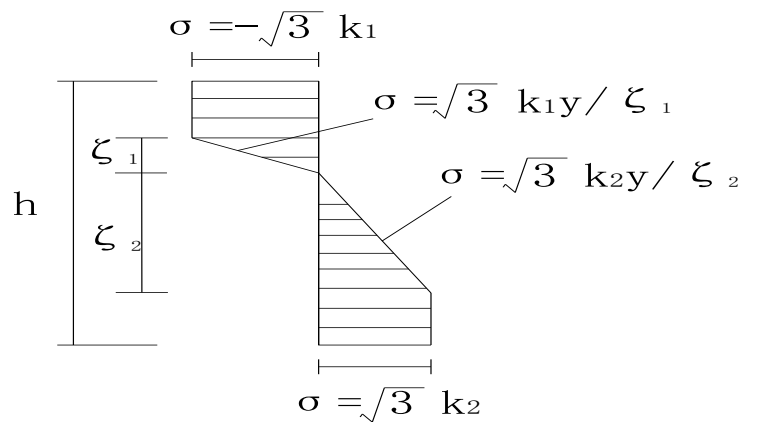

Fig.11. Stress distribution during the elastic-plastic period.

$$
\frac{E_{c} \xi_{1}}{\rho}=\sqrt{3} k_{1} \text { and } \frac{E_{t} \xi_{2}}{\rho}=\sqrt{3} k_{2}
$$

Then we have

$$
\xi_{1}=\frac{\sqrt{3} k_{1} \rho}{E_{c}} \text { and } \xi_{2}=\frac{\sqrt{3} k_{2} \rho}{E_{t}}
$$

That is

$$
\frac{\xi_{1}}{\xi_{2}}=\frac{k_{1}}{k_{2}} \frac{E_{t}}{E_{c}}
$$

Therefore, $\xi 1 / \xi 2$ is a constant. When $\xi 1 \rightarrow 0$ or $\xi 2 \rightarrow 0$, the tensile region and compressive region reach saturated simultaneously.

The plastic development of the bi-modulus beam is divided into four periods, including the elastic period, the first elastic-plastic period, the second elastic-plastic period and the plastic period.

(1) The elastic period

During this period, the maximum tensile stress and compressive stress are both less than the control stress. The moment of the bi-modulus beam is

$$
M=\frac{\left(E_{c} h_{1}{ }^{3}+E_{t} h_{2}{ }^{3}\right) b}{3 \rho}=-\frac{\left(E_{c} h_{1}^{3}+E_{t} h_{2}^{3}\right) b}{3} \frac{d^{2} v}{d x^{2}}
$$

The concentrated force on the bi-modulus beam is

$$
P \leq\left[\frac{\sqrt{3} k_{1} \sqrt{\alpha} b h^{2}}{3(1+\sqrt{\alpha})\left(L-L_{1}\right)}, \frac{\sqrt{3} k_{2} b h^{2}}{3(1+\sqrt{\alpha})\left(L-L_{1}\right)}\right]_{\min }
$$

(2) The first elastic-plastic period

The plastic region starts to develop at either the top region or the bottom region of the cross section of the bi-modulus beam, while another region still keeps in elastic period. The plastic region is identified according to $\alpha$ and $\mathrm{k} 1 / \mathrm{k} 2$.

When $\frac{k_{1}}{k_{2}}<\frac{1}{\sqrt{\alpha}}$, the plastic region will firstly develop at the top region, while the bottom region will stay in elastic period. And we have

$$
\begin{gathered}
M=\frac{\sqrt{3} k_{1}\left(3 h_{1}^{2}-\xi_{1}^{2}\right) b}{6}-\frac{E_{t} b h_{2}^{3}}{3} \frac{d^{2} v}{d x^{2}} \\
\frac{\sqrt{3} k_{1} \sqrt{\alpha} b h^{2}}{3(1+\sqrt{\alpha})\left(L-L_{1}\right)}<P<\frac{\sqrt{3} k_{2} b h^{2}}{3(1+\sqrt{\alpha})\left(L-L_{1}\right)}
\end{gathered}
$$


When $\frac{k_{1}}{k_{2}}>\frac{1}{\sqrt{\alpha}}$, the plastic region will firstly develop at the bottom region with the top region keeping elastic state. And we have

$$
\begin{gathered}
M=\frac{\sqrt{3} k_{2}\left(3 h_{2}^{2}-\xi_{2}^{2}\right) b}{6}-\frac{E_{c} b h_{1}^{3}}{3} \frac{d^{2} v}{d x^{2}} \\
\frac{\sqrt{3} k_{1} \sqrt{\alpha} b h^{2}}{3(1+\sqrt{\alpha})\left(L-L_{1}\right)}<P<\frac{\sqrt{3} k_{2} b h^{2}}{3(1+\sqrt{\alpha})\left(L-L_{1}\right)}
\end{gathered}
$$

However, if $\frac{k_{1}}{k_{2}}=\frac{1}{\sqrt{\alpha}}$, the plastic region will develop at both the top and bottom region simultaneously. The plastic development will go straightly into the second elastic-plastic period with the first plastic period omitted and we have

$$
P=\frac{\sqrt{3} k_{2} b h^{2}}{3(1+\sqrt{\alpha})\left(L-L_{1}\right)}=\frac{\sqrt{3} k_{1} \sqrt{\alpha} b h^{2}}{3(1+\sqrt{\alpha})\left(L-L_{1}\right)}
$$

(3) The second elastic-plastic period

Both the top region and bottom region will go into plastic state but the whole region is underdeveloped. Then we have

$$
\begin{gathered}
M=\frac{\sqrt{3} k_{1} b\left(3 h_{1}^{2}-\xi_{1}^{2}\right)+\sqrt{3} k_{2} b\left(3 h_{2}^{2}-\xi_{2}^{2}\right)}{6} \\
P \geq\left[\frac{\sqrt{3} k_{2} b h^{2}}{3(1+\sqrt{\alpha})\left(L-L_{1}\right)}, \frac{\sqrt{3} k_{1} \sqrt{\alpha} b h^{2}}{3(1+\sqrt{\alpha})\left(L-L_{1}\right)}\right]_{\text {max }}
\end{gathered}
$$

(4) The plastic period

Under the ideal condition, the plastic region develops completely in the bi-modulus beam and the structure will be destroyed finally. The moment can be described as below

$$
M=\frac{\sqrt{3} k_{1} b h_{1}^{2}}{2}+\frac{\sqrt{3} k_{2} b h_{2}^{2}}{2}
$$

or

$$
M=P \times\left(L-L_{1}\right)
$$

Combine Eq.(18) and Eq.(19), and then the ultimate load can be obtained

$$
P_{0}=\frac{\sqrt{3} b\left(k_{1} h_{1}^{2}+k_{2} h_{2}^{2}\right)}{2\left(L-L_{1}\right)}
$$

\section{Structural Experiment}

Experimental analysis during the elastic-plastic period is conducted according to the same tests in Section 3.1.

\section{Comparison of Theoretical and Experimental Results}

Take as examples the specimens in the experiments and obtain the $\mathrm{N}-\varepsilon$ curve at different test points using the elastic-plastic bi-modulus theory in Section 4.1. The comparison of the theoretical results with the experimental results is shown in Figure.12. 


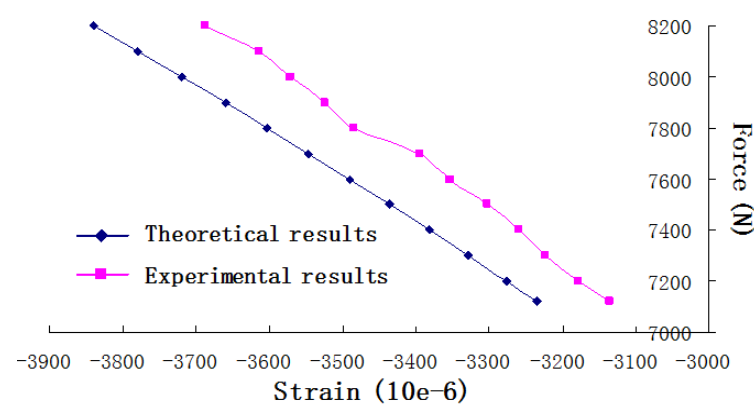

(a). Test point (No.1).

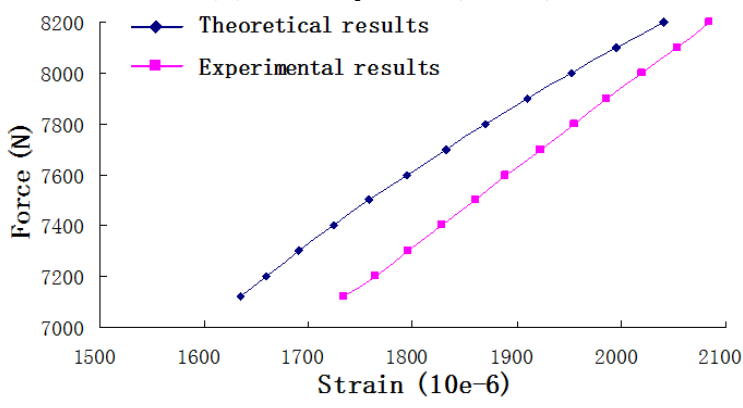

(c). Test point (No.4).

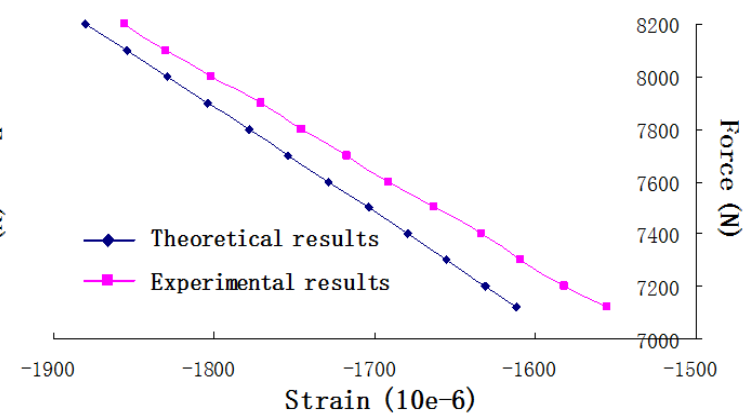

(b). Test point (No.2).

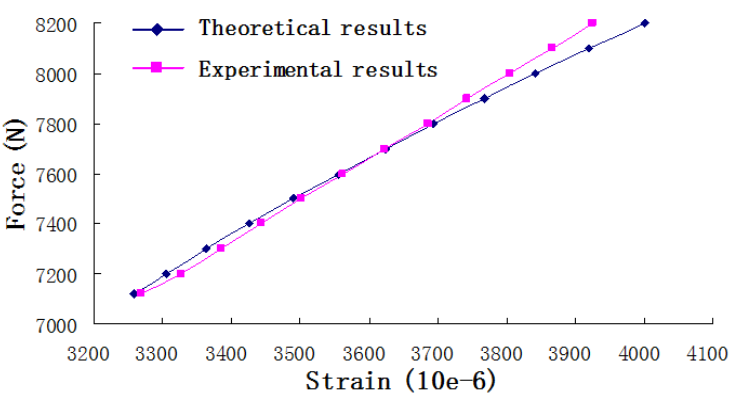

(d). Test point (No.5).

Fig.12. N- $\varepsilon$ curve during the elastic-plastic period.

It is shown that the elastic-plastic analysis method proposed in this paper can describe the elasticplastic development in bi-modulus beam accurately and rationally.

\section{Conclusions}

In this paper, material tests, structural experiment and the elastic-plastic analysis of bi-modulus graphite beam are conducted. The conclusions in details are as below:

(1) Graphite is a kind bi-modulus material, with $E_{c} / E_{t} 1.41$ approximately.

(2) The strain in the tensile region is higher than the stain in the compressive region, which indicates that the neutral axis deflects towards the compressive region. Simultaneously, the deflection increases with the increase of the load.

(3) Comparing with results of the structural experiment, it is verified that the analytical solution of bending bi-modulus beam subjected to lateral force in [10] is accurate and rational.

(4) Comparing with results of the structural experiment, it is verified that the elastic-plastic analysis method proposed in this paper is rational and reliable.

(5) The plastic mechanical law of the simple supported bi-modulus beam is listed as below,

a) During the elastic period, as the external force increases, the position of neutral axis keeps constant. During the plastic period, with the external force increasing, the neutral axis moves to the top surface of the cross section. The final position of the neutral axis is determined according to the ratio of tensile yield limit and compressive limit.

b) Under the condition that the tensile yield stress and compressive yield stress keep constant, with the increase of the Et/Ec, the first elastic-plastic region extends and the elastic region and second elastic-plastic region contract.

c) During the elastic period, Et/Ec has a relatively strong effect on the stress distribution. The maximum tensile stress can be decreased by decreasing Et/Ec and the maximum compressive stress can be decreased by increasing Et/Ec. However, after the plastic region develops in the bi-modulus beam, the effect of Et/Ec on the stress distribution decreases.

d) With the plastic region extending in the bi-modulus beam, the effect of Et/Ec on the strain distribution does not decrease. Therefore, the maximum of tensile strain and compressive strain can be changed by changing Et/Ec. When Et/Ec is more than 0.5 , the maximum of tensile strain and compressive strain will increase as Et/Ec increases during the elastic-plastic period. 


\section{References}

1. Z.H. Guo, X.Q. Zhang, ACI Mater. J. 84, 278-285 (1987)

2. G.N.J. Gilbert, Brit. Cast Res. Assn. J. 9, 347-363 (1961)

3. G.A. Medri, J. Eng. Mater. Technol. 26, 26-28 (1982)

4. K. Cai, Z. Luo, Q.H. Qin, Eng. Comput. 31, 1361-1378 (2014)

5. S. Mohanty, S. Majumdar, M. Srinivasan, Nucl. Eng. Des. 260, 145-154 (2013)

6. C. Park, AIAA J. 14, 1640-1642 (2015)

7. T.R. Prabhu, V.K. Varma, S. Vedantam, Wear 309, 1-10 (2014)

8. T. Lin, L. Zhong, J. Wang, L. Guo, H. Wu, Q. Guo, F. Fu, G. Chen, Biosens. Bioelectron. 59, 89-93 (2014)

9. J.L Zeng, S.H. Zheng, S.B. Yu, F.R. Zhu, J. Gan, Appl. Energy 115, 603-609 (2014)

10. W.J. Yao, Z.M. Ye, J. Appl. Math. Mech. (Engl. Transl.) 25, 983-993 (2004) 